\title{
Diagnosis of Liver Tumor from CT Images Using First Order Statistical Features
}

\author{
Dr. Alyaa H. Ali ${ }^{\# 1}$, Entethar M. Hadi ${ }^{* 2}$ \\ ${ }^{1}$ Dr. Alyaa Hussein Ali/ Baghdad University/ College of Science for Women, Iraq. \\ ${ }^{2}$ Entethar Malek Hadi is currently pursuing masters degree program in Science for Women-University of Baghdad in physics (Digital Image \\ Processing), Iraq.
}

\begin{abstract}
The detection and diagnose of liver tumors from CT images by using digital image processing, is a modern technique depends on using computer in addition to textural analysis to obtain an accurate liver diagnosis, despite the method's difficulty that came from liver's position in the abdomen among the other organs. This method will make the surgeon able to detect the tumor and then easing treatment also it helps physicians and radiologists to identify the affected parts of the liver in order to protect the normal parts as much as possible from exposure to radiation. This study describes a new 2D liver segmentation method for purpose of transplantation surgery as a treatment for liver tumors. Liver segmentation is not only the key process for volume computation but also fundamental for further processing to get more anatomy information for individual patient. Due to the low contrast, blurred edges, large variability in shape and complex context with clutter features surrounding the liver that characterize the CT liver images. In this paper, the CT images are taken, and then the 2D segmentation process which is based on the hybrid method which is the combination of modified $k$ Mean (which depend on the distance and color), the statistical structure which is the first order statistical feature are applied to the liver image which will find, extract the CT liver boundary and further classify liver diseases.
\end{abstract}

Keywords - Computed Tomography (CT), Modified K-mean, Irregularity.

\section{INTRODUCTION}

According to recent statistics, liver cancer is one of leading cancerous diseases in Iraq. Thus far, the only definitive test for liver cancer is needle biopsy. However, the needle biopsy is an invasive technique and generally not recommended unless there is an absolute need [1]. To avoid unnecessary needle biopsy, doctors can take advantage of information provided by images acquired from various medical imaging systems such as surface texture, object boundary extraction, tumor detection, etc. to assist them to improve their diagnosis [2]. In this case, an effective image analysis is important. Although many segmentation methods have been successfully used in medical imaging, it has found that most of them generally do not perform well in segmenting the liver boundary from a CT liver image [3]. This is mainly due to the fact that there are other organs adjacent and close to the liver which makes segmentation more difficult. In addition, the liver itself may also contain tumors such as hepatoma and haemagioma that can obscure the boundary [4]. In order to address these problems, we present an automatic CT liver image classification system which can be used to detect four types of liver tumor: hepatoma, haemagioma, cyst and cirrhosis. The aim of this work is to segment a tumor in a liver; this will make the surgeon able to see the tumor and then will be easy for the treatment.

In this study, the CT images are taken, and then the segmentation processes are applied to the liver image which will find, extract the CT liver boundary and further classify liver diseases.

\section{MASK CREATION}

Mask Creation is used to create the binary mask which has the value of 0 or 1's. This operation is performed by Specify polygonal region of interest (ROI) in MATLAB software [5]. This is used to draw around the edge of the liver manually, making it possible to discard all irrelevant information. Here we can see the result of our own encirclement of the liver:

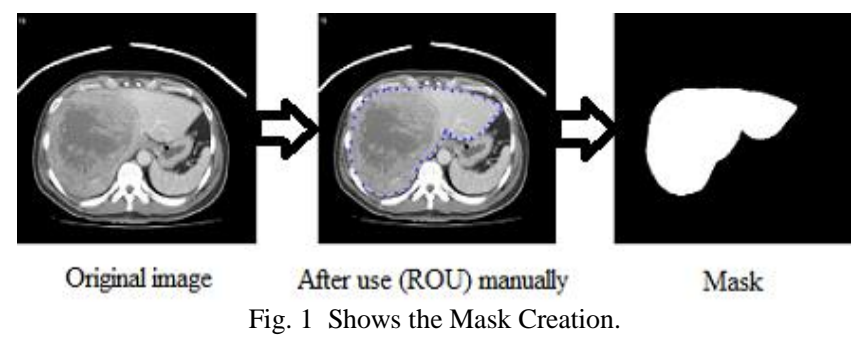

A binary image used as a mask. For masked filtering where the liver is giving the value 1 and the background is given the value 0 , the final step involved applying the segmented mask in Fig. 1 to the original image (Fig. 1), multiplying the final eroded image with the original image to give the masked image. Colors play an important role for object detection, tracking and recognition, etc. The problem of RGB (Red, Green, and Blue) does not provide the correct information about liver color due to the problem of X-ray effects and the presence of other organs of similar density to the liver, so we converted the masked image to HSV color space this can be seen in Fig. 2.

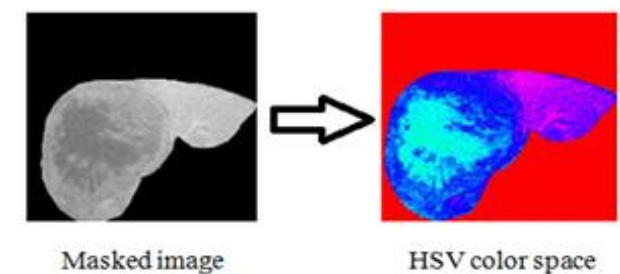

Fig. 2 Shows converted the masked image to HSV color space. 


\section{Proposed ALGORITHM FOR CT CLASSIFICATION}

The basic principle of the proposed algorithm is integrating the contour with K-means clustering.

1) Read the HSV image.

2) Apply the decorrelation stretching for color separation.

3) Convert original image from HSV color space type to $\mathrm{L} * \mathrm{a} * \mathrm{~b} *$ color space.

4) Apply modified K-Means Clustering method which depends on the color and distance for each sub image of $a^{*} b^{*}$ bands.

5) Depending on the result of clustering, label every pixel in the image.

6) Create new segmented images depending on color.

7) Depending on the label pixel separate objects in image by color.

8) Output the segmentation nuclei into a Separate Image. Then programmatically determine the index of the cluster containing the blue objects because $\mathrm{K}$-means will not return the same cluster index value every time. We can do this using the cluster center value, which contains the mean ' $a{ }^{*}$ ' and ' $b$ *' value for each cluster. This process can be seen in Fig. 3.

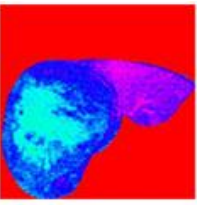

Original Image

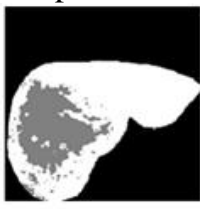

Grayscale image

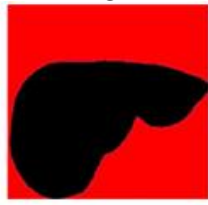

The background

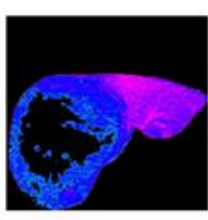

Healthy part

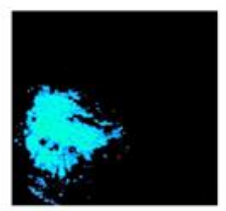

Tumor part
Fig. 3 Shows the segmentation result

The K-mean clustering segmented the images depending on the distance and the color feature which is a modified $\mathrm{K}$-mean. The traditional K-mean depends only on the distance between the centroid while the modified K-mean depends on both the distance and color. The result aims to developing an accurate and more reliable image which can be used to help the physicians for medical diagnosis which can be used in locating liver tumors, measure tissue volume and in more. The advantage of K-means algorithm is simple and quite efficient. It works well when clusters are not well separated from each other. There are different types of tumors in liver, may be as Hepatoma, Haemangioma, Cyst, or Cirrhosis. Suppose if it is a tumor, then $\mathrm{K}$-means algorithm is to extract it from the liver cells. If there is any noise present in the CT image, it is removed before the K-means process. The noise free image is given as input to the K-means and tumor is extracted from the CT image, then segmentation using thresholding of output in feature extraction. Finally, approximate reasoning for calculating tumor shape. The flowchart of histogram clustering and the k-means clustering is as shown in the Fig. 4.

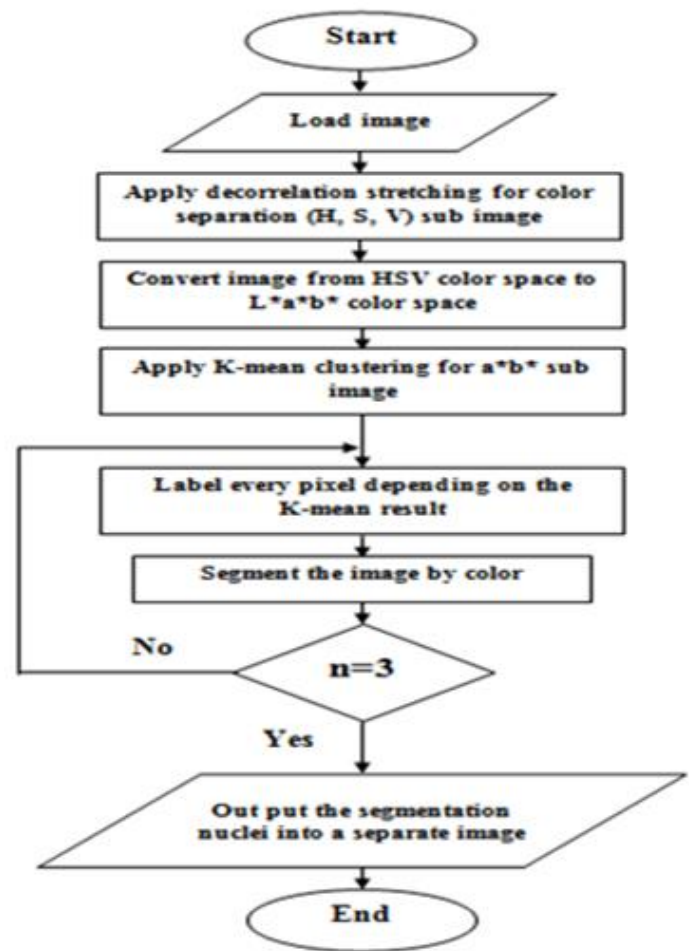

Fig.4 Shows the flowchart of histogram and k-means clustering.

\section{IMAGE SEGMENTATION USING K-MEAN}

We take two different cases for each of the Hepatoma and Haemangioma. We have implemented K-means clustering algorithm. Which is unsupervised clustering of images based on the color features. This process helps us to distinguish the normal liver tissue from the abnormal. See figure (5) and figure (6) shows this process. Also it helps us to distinguish between different types of liver tumors such as malignant tumor and benign tumor from the determination of the statistical features for each class like as entropy, Mean, Standard deviation, Energy, Skewness and Kurtosis, Then comparing with the normal and abnormal part of liver.

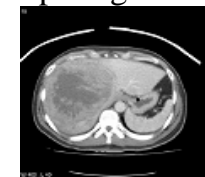

Original image No.1

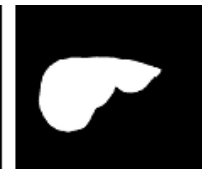
Mask

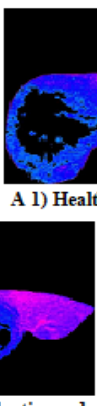

2) Healthy

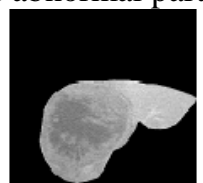

Liver

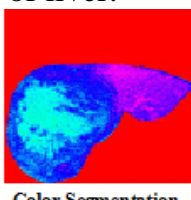

Color Segmentation

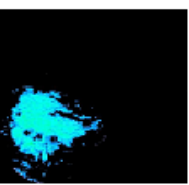

A 4) Hepatoma

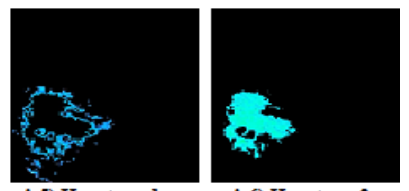

A 5) Hepatomal
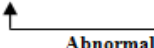

Fig. 5 Shows the original image of hepatoma with normal and abnormal part. 


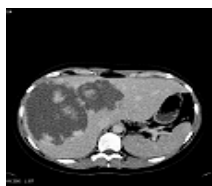

Original image No.2

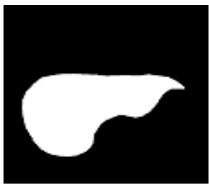

Mask

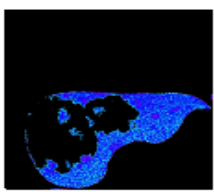

G 1) Healthy tissues
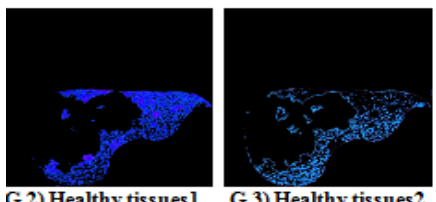

$\uparrow$ 个 Normal .

Fig. 6 Shows the original image of haemangioma with normal and abnormal part.

We have taken one cases of the healthy liver in order to compare these cases with other abnormal cases. This can be seen in Fig. 7.
Original image No.3

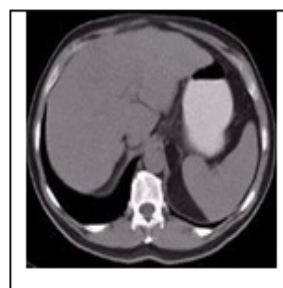

Mask

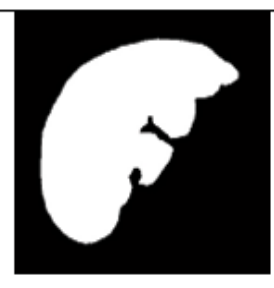

Liver

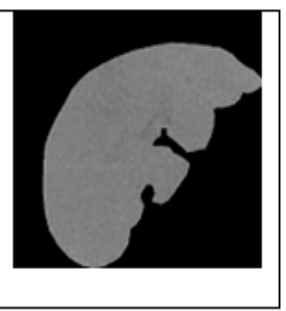

Fig. 7 Shows a healthy liver Image.

\section{TEXTURES FEATURES FOR NORMAL AND} ABNORMAL CASES

When all the textures analysis of the image is completed, we take the average value for all cases Healthy liver, Hepatoma and Haemangioma, and then the calculation of its average would be clear by viewing it in tables form.

TABLE 1 AVERAGE VALUE FOR TEXTURE FEATURES FOR ALL CASES (NORMAL AND ABNORMAL LIVER).

\begin{tabular}{|l|c|c|c|c|c|c|}
\hline \multicolumn{1}{|c|}{ Class No. } & Entropy & Mean & $\begin{array}{c}\text { Standard } \\
\text { Dev. }\end{array}$ & Energy & Skewness & Kurtosis \\
\hline Healthy liver & 0.43175 & 60.84065 & 68.69436 & 0.29195 & $4.74 \mathrm{E}-06$ & $1.03 \mathrm{E}-09$ \\
\hline Hepatoma & 0.41174 & 67.6777 & 80.60463 & 0.28264 & $3.16 \mathrm{E}-06$ & $5.24 \mathrm{E}-10$ \\
\hline Haemangioma & 0.37764 & 56.09866 & 72.77617 & 0.39864 & $5.38 \mathrm{E}-06$ & $1.22 \mathrm{E}-09$ \\
\hline
\end{tabular}

From the table (1) we can see that the Hepatoma has the highest value for the Mean that is the Hepatoma is brighter than the others. As well as the Kurtosis for the Hepatoma case has the lowest value compare with the others. The Haemangioma has the largest value of energy, this means that the Haemangioma is regular in it textural and has less gray value.

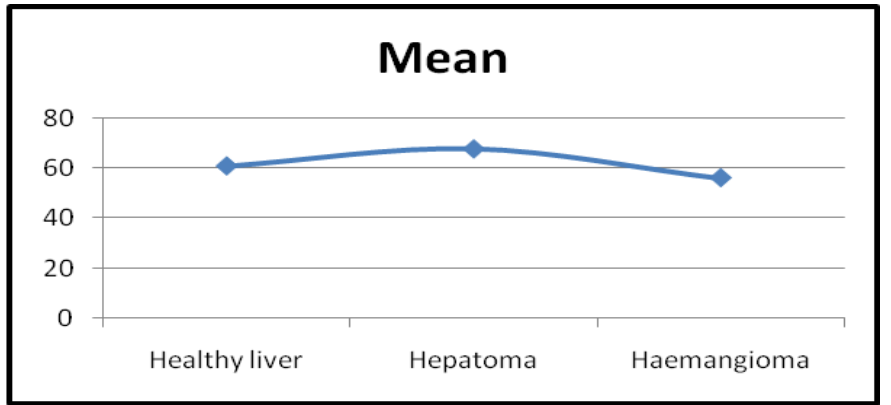

Fig. 8 Shows the graph of the Mean of different cases in table (1).

Mean value for the Hepatoma is highest than the others, because the Hepatoma is more bright than the others cases.

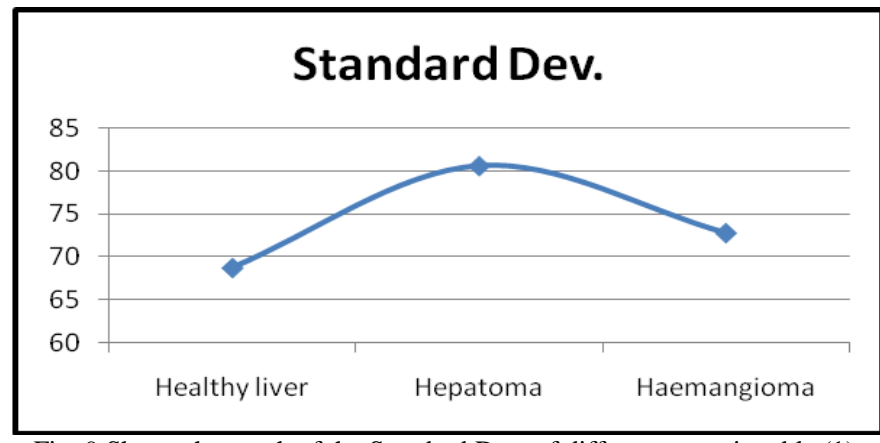

Fig. 9 Shows the graph of the Standard Dev. of different cases in table (1)

Also the standard deviation is high for the Hepatoma, because it's brighter than the other cases.

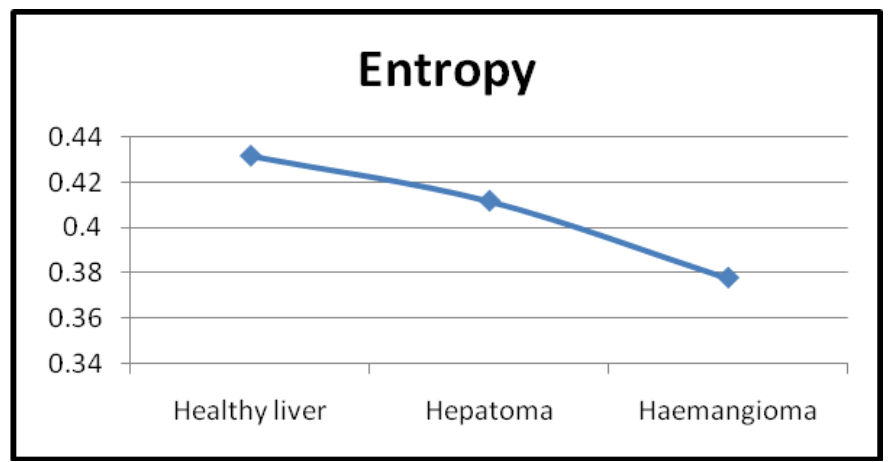

Fig. 10 Shows the graph of the Entropy of different cases in table (1)

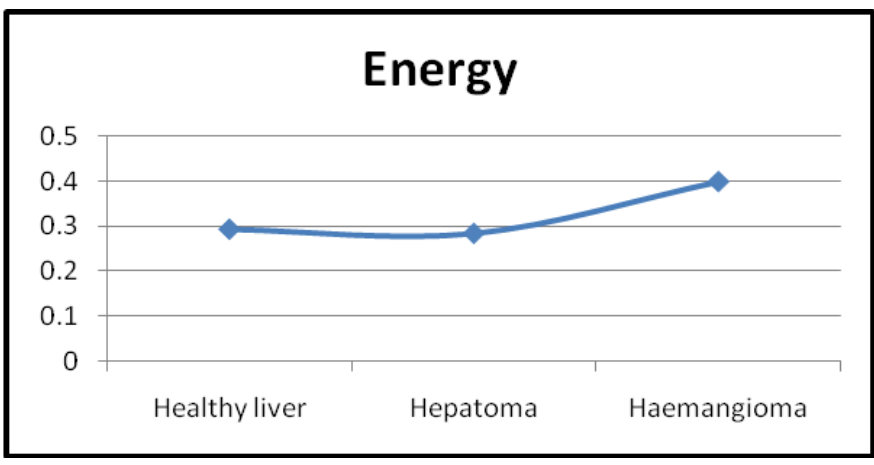

Fig. 11 Shows the graph of the Energy of different cases in table (1). 
Healthy liver gives the highest value in Entropy, this means it has less Energy value since the Energy inversely proportional to the Entropy. This means that the healthy liver has great number of gray level. We can show that in Fig. 10 and Fig. 11.

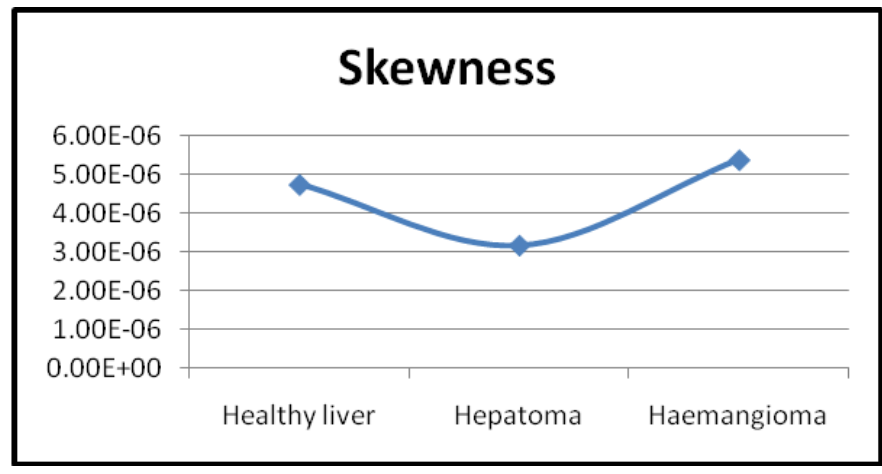

Fig. 12 Shows the graph of the Skewness of different cases in table (1).

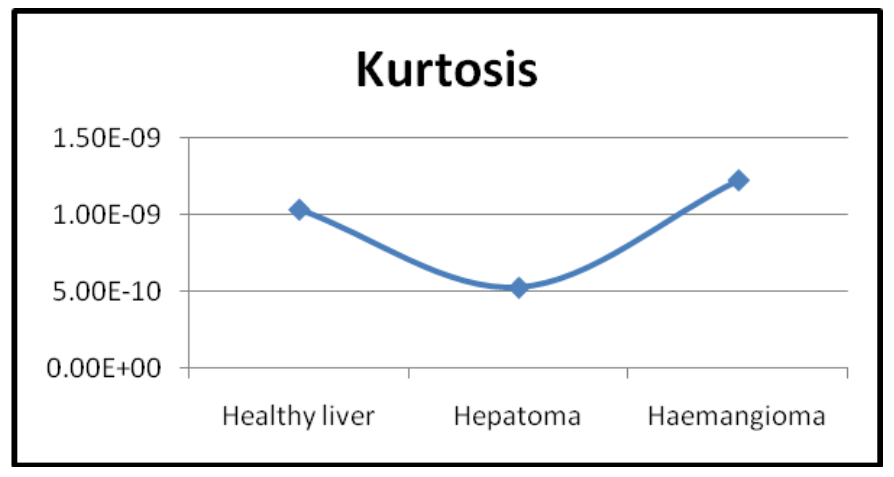

Fig. 13 Shows the graph of the Kurtosis of different cases in table (1).

In Fig. 12 the Skewness gives indication about the symmetry in the texture. So the Hepatoma has the lowest value, it has less symmetry in the texture. As well as for
Kurtosis in Fig. 13. This means that the texture is not regular and non homogenous.

\section{CONCLUSIONS}

In medical field the doctors go for a manual technique to detect and locate the liver tumor, here is an approach to detect the tumor automatically. Tumor detection using CT image has been done using the digital image processing. We have developed an automated method for the detection of tumors in liver CT images using Mask, color map, color segmentation, modified K-means clustering and image processing techniques. The segmentation accuracy is obtained using the modified $\mathrm{K}$ means clustering. Our system has been successfully tested on a large number of tumor images, liver tumor implemented for the discrimination of the normal and pathological tissues. The liver regions related to a tumor can be exactly separated from the liver image. The statistical properties like Mean, Variance, Standard deviation, Skewness, Kurtosis, Energy and Entropy have been calculated for liver tumor, which helps in the diagnosis of the type of tumor that was malignant or benign. This system can be applied to detect tumor-like growth much before they are visible to the human eye.

\section{REFERENCES}

[1] Upadhyay, Y. and Wasson, V. 2014. "Analysis of Liver MR Images for Cancer Detection using Genetic Algorithm". International Journal of Engineering Research and General Science. Vol.2, No.4, PP: 730-737.

[2] Kumar, P. Bhalerao, S. 2014. "Detection of Tumor in Liver Using Image Segmentation and Registration Technique". IOSR Journa of Electronics and Communication Engineering (IOSR-JECE). Vo.9, No.2, PP: 110-115.

[3] Selle, D.; Spindler, W.; Preim, B. and Peitgen, H. O. 2000 "Mathematical Methods in Medical Imaging: Analysis of Vascular Structures for Liver Surgery Planning". PP: 1-21.

[4] Zimmer, C. and Olivo-Marin, J. C. 2005. "Coupled Parametric Active Contours". Transactions on pattern Analysis and Machine Intelligence. Vol.27, No.11, PP: 1838-1841.

[5] Chitra, S. and Balakrishnan, G. 2012. "Comparative Study for Two Color Spaces HSCbCr and YCbCr in Skin Color Detection". Applied Mathematical Sciences. Vol.6, No.85, PP: 4229 - 4238. 